\title{
Cloud and Radiation Mission with Active and Passive Sensing from the Space Station
}

\author{
James D. Spinhirne \\ Laboratory for Atmospheres, NASA Goddard Space Flight Center, Greenbelt, MD 20771 \\ [301-286-9099, spinhirne@gsf.nasa.gov]
}

\begin{abstract}
A cloud and aerosol radiative forcing and physical process study involving active laser and radar profiling with a combination of passive radiometric sounders and imagers would use the space station as an observation platform. The objectives are to observe the full three dimensional cloud and aerosol structure and the associated physical parameters leading to a complete measurement of radiation forcing processes. The instruments would include specialized radar and lidar for cloud and aerosol profiling, visible, infrared and microwave imaging radiometers with comprebensive channels for cloud and aerosol observation and specialized sounders. The low altitude. available power and servicing capability of the space station are significant advantages for the active sensors and multiple passive instruments.
\end{abstract}

\section{INTRODUCTION}

Since clouds and aerosol play an overwhelming role in the climate system, it is generally recognized that the lack of knowledge of the cloud and aerosol leads to one of the main uncertainties for the prediction and modeling of climate. As a result, cloud and aerosol observations are generally placed as the top priority for climate research, for example by the U.S. Global Change Research Program and Intergovernment Panel on Climate Change. Current satellite programs are addressing the needs of cloud and aerosol observations. For example, ISCCP (International Satellite Cloud Climatology Project) retrieves global cloud statistics from existing satellite data. Cloud and aerosol observations are to be significantly improved by the EOS satellites to be launched in the next few years. International satellites such as ADEOS include special capabilities for cloud and aerosol observations.

Despite a seeming plethora of existing and planned satellite cloud observations, it is well understood that our knowledge of clouds is still to be limited by our inability to resolve the full three-dimensional structure of clouds and other important variables on a global scale. The influence of clouds on the vertical distribution of radiative heating in the atmosphere and the distribution of radiative fluxes are crucial areas that are equal in importance to latent heating. The vertical distribution of cloud water and cloud ice must be known globally.

Also the magnitude and distribution of radiative influence by aerosol is recognized to be a major uncertainty for present modeling of climate. Aerosols in the atmosphere are highly variable. Transport and radiative heating is related to the vertical distribution. In addition to the vertical distribution, radiation effects are dependent on aerosol absorption. These factors are not to be sufficiently observed within existing programs. Improved future space observations are needed to adequately present answers to the following questions:

1. What is the vertically distribution of radiation heating and cooling due to clouds? What is the influence of multiple cloud layering?

2. What is the amount of liquid and frozen water that the hydrological cycle moves into and through the atmosphere?

3. What is the diurnal variation in cloud cover, and what is the diurnal measurement bias of current cloud sensing?

4. What is the climatology for the aerosol loading in the atmosphere? What is the height distribution of aerosol and its seasonal dependence? What are the sources and sinks?

5. What are the typical values for aerosol radiative heating or negative radiation forcing and aerosol absorption and scattering properties on which they depend; how are they distributed and how do they vary? 
We will describe a comprehensive remote sensing experiment combining active and passive observations to address these questions. The central feature of the experiment is the use of active radar and lidar profiling in combination with comprehensive passive measurements. Use of the International Space Station as a platform for the experimental observations is appropriate due to the requirements for the active sensors and also the potential to obtain a combination of measurement from low cost instruments.

\section{MEASUREMENT APPROACH AND SPECIFIC OBJECTIVES}

A combination of passive sensing and active laser and radar sensors for profiling the vertical structure of the atmosphere is needed to address the issues raised above. The passive sensing involves an appropriate combination of visible, UV, IR and microwave radiometry with required polarization and multiple angle sensing included for visible and UV wavelengths. The passive observations would be made with cloud radar and cloud and aerosol lidar sensing that can fully profile the cloud and aerosol structure of the atmosphere. The combination of instruments would be focused on the specific science questions as listed above.

The objective of the mission for clouds would be the retrieval full three dimensional structure of clouds and cloud radiative heating and cooling along with quantitative retrieval of the amount of liquid water, water vapor and ice in clouds and microphysics important to radiation and cloud processes. Full vertical profiling of clouds requires both radar and lidar (Brown et al., 1995; Spinhirne et al., 1996). Cloud profiling radar can define the structure of dense, optically thick clouds but lacks the sensitivity to adequately detect thin clouds. Lidar does not profile into dense clouds beyond the limits of optical attenuation but is very sensitive to all thin clouds. There are a variety of techniques for the retrieval of cloud physics parameters which involve visible and infrared multispectral imagery. Many of these are improved with the addition of direct measurements of the height structure of clouds. The high spectral infrared measurements of Fourier transform spectrometers (FTS) may be combined with direct cloud detection for better sounding and cloud microphysics retrievals. The amount of liquid and frozen water in the atmosphere can be approached with a combination of microwave radiometer frequencies. A recently developed technique uses very high frequencies in the 300 to $800 \mathrm{GHz}$ ranges for retrieval of the ice content of clouds. Water vapor can also be retrieved in cloudy and cloud free regions from microwave sounding. To put any cloud retrievals in context, it is important that measurements be combined with broadband radiometer measurements of top of the atmosphere flux.

The experiment would make a comprehensive measurement of the distribution and radiative properties of aerosol. Lidar can accurately and sensitively profile the vertical structure of aerosol scattering. A limitation is that the variable directly observed by backscatter lidar is weakly related to aerosol radiation parameters. Passive sensing has other limitations. Retrieval of aerosol optical depth (aod) by near infrared reflectance is limited to low surface albedo and thus ocean regions only. There are also techniques utilizing UV wavelengths and polarization. Currently TOMS detects the presence in the amosphere of UV-absorbing aerosols (smoke, dust, volcanic ash) and non-absorbing aerosols (mostly sulfates) in the UV-wavelength range 331 to $380 \mathrm{~nm}$ over both land and water (dark background in the UV). This conversion can be done accurately only if the height of the aerosol cloud is known. Polarization analysis also removes sensitivity from surface albedo and can be used in a retrieval of aod and also an aerosol particle size parameter. The correct mix of passive measurements and lidar would provide for comprebensive sensing of aerosol parameters and would be provided by the proposed mission.

\section{Instruments}

The suggested measurement and instrument suite for the cloud and aerosol remote sensing mission are summarized in table 1 and individually described below.

\section{Cloud Radar}

Cloud profiling radar is a relatively new instrument technique, but one that is well demonstrated. cloud radar at 95 $\mathrm{gHz}$ will profile through thick clouds and indicate cloud base in many cases, but it is to be expected that thin clouds, especially when composed of small particles, will not be observable. Although cloud radar directly shows vertical structure, the signal depends in a complex way on cloud microphysics and cannot independently provide 
quantitative variables. A sensitive cloud radar is a large instrument requiring significant power.

\section{Cloud and Aerosol Lidar}

For atmospheric lidar, large, instruments are required to obtain sufficient performance for basic cloud and aerosol profiling. Another limitation is that the basic measurement of these instruments is a quantity, the attenuated backscatter that is not directly related to the important physical and radiation parameters of clouds and aerosols. An advanced lidar concept involving multiple wavelengths and fields-of-view for cloud and aerosol observations can address the two issues given above. CW diode pumped, high pulse frequency lasers, and the use of narrow band tuned etalon blocking filters will improve the efficiency and coverage of spaceborne cloud and aerosol profiling. Lidar is sensitive to thin cloud and aerosol but does not profile through thick clouds. In combination with radar, full cloud profiling is possible. Also in some cases, combined signals from radar and lidar are applicable to particle size retrieval .

\section{Visible and IR Imaging Radiometer}

Imaging radiometers at visible and infrared wavelengths are a basic instrument for measurements of sea surface temperature, cloud coverage, vegetation and aerosols. There are benefits to earth remote sensing from increasing spatial resolution, the number of bands and the spectral resolution of bands. Instruments based on array detectors, such as the imagers on the Lewis satellite and the shuttle ISIR instrument lead in this direction.

\section{Broadband Radiometers}

Radiative flux measurement is a requirement for any experiment relating to cloud forcing. The CERES instrument on EOS is an example. A more capable and lower cost instrument could possibly be developed.

\section{UVNis Aerosol Radiometer}

Shortwave instruments for aerosol and some cloud retrievals, such as those from TOMS, are making rapid progress in application techniques and instrumentation. For new UV sounding, the spatial resolution can be improved to $1 \mathbf{x}$ $1 \mathrm{~km} 2$ from the current TOMS average pixel of $100 \times 100 \mathrm{~km} 2$. This would enable viewing between what are now sub-pixel clouds known to be present in otherwise clear scenes. The UV aerosol absorption retrievals rely on knowledge of the aerosol vertical profile. Currently the height distribution is estimated. The combination of UV and lidar sounding can provide a three dimensional quantitative measurement of aerosol radiative forcing parameters.

\section{Polarization and Bi-directional Instruments}

The CNES Polder instrument has shown the value of the combination of polarization and wide FOV bi-directional measurements in an array detector instrument

TABLE 1 Measurement summary

\begin{tabular}{|c|c|}
\hline Measurement & Application \\
\hline Cloud Radar & Profile Dense Clouds \\
\hline Multi Channel Cloud and Aerosol Lidar & $\begin{array}{c}\text { Profile Thin Clouds and Aerosol } \\
\text { Direct Optical Thickness Measurement }\end{array}$ \\
\hline Multiwavelenth Visible and IR Radiometer & Cloud/Aerosol Distribution Particle Retrievals \\
\hline Broadband Radiometers & TOA Flux \\
\hline UV, Bi-directional and Polarization Shortwave \\
Measurements & $\begin{array}{c}\text { Aerosol Single Scatter Albedo, Optical Thickness, } \\
\text { Scattering Function, Cloud Particle Characteristics }\end{array}$ \\
\hline Microwave Radiometer & Liquid/lce Water Content \\
\hline FTS & Particle Retrieval, Sounding, IR Forcing \\
\hline
\end{tabular}

for aerosol and cloud remote sensing. High precision polarization measurement by a compact scanning photopolarimeters is a technology that can be rapidly advanced.

Microwave Radiometer. The use of low frequency microwave sensing to retrieve cloud liquid water content over oceans is well known. In the past few ycars theoretical and observational studies have indicated that high frequency radiometer channels above $300 \mathrm{GHz}$ have applications for the water content of ice crystal clouds. Advances in technology allow radiometers with chinnels to $800 \mathrm{GHz}$. 
FTS. The infrared sounding potential of FTS instruments has applications for cloud particle retrievals which is significantly enhanced if combined with direct cloud vertical structure information.

\section{MISSION CHARACTERISTICS}

There are a number of reasons why the International Space Station should be considered as a platform for the multi sensor cloud sensing mission. Most basically, an independent free flyer mission including the instrument and measurement suite described above would be extremely expensive due to the number of instruments and the power and mass required. A well demonstrated alternative to the difficulty and expense of typical free satellite instruments are Space Shuttle Hitchhiker (SSH) experiments. SSH instrument have been successfully flown for costs on the order of a tenth that of an equivalent free flyer instrument. Part of the cost savings relates to the low lifetime needed for shuttle experiment, but to a larger extent the advantages stem from a standard and easy to implement data, control, power and thermal interface environment. In addition size, power and mass are not as significantly limited as for a free satellite. It is to be expected that the $\mathrm{SSH}$ advantages can also be the case for station experiments. A possible implementation is a facility to directly transfer SSH instruments to the station. In addition the capability for repair and replacement is also a major advantage over instruments on independent satellites.

The International Space Station will be an especially advantageous platform for active sounding instruments. The operation of all such instruments are limited by the transmit/receive power-aperture product. An example of a basic lidar profiling instrument is the EOS GLAS system. To obtain even the $300 \mathrm{~kg}$ and $300 \mathrm{w}$ mass and power requirements of the instrument has required costly engineering. For other studies and more advanced measurements, power requirements generally approach kilowatts and large apertures significantly increase mass. The situation is similar for cloud radar where measurement sensitivity is a significant issue. The available power and mass to be expected for instruments transferred from shuttle to station should significantly ameliorate power and mass limits. Instrument lifetime is equally an advantage for the station platform. High powered lasers with multiple year lifetimes are very difficult to engineer compared to engineering for replacement.

A critical issue is whether the space station orbit is appropriate for a cloud and aerosol radiative forcing mission. For several considerations, the orbit is advantageous. The low orbit altitude is an advantage for active sounding instruments. Required power-aperture product is reduced in general by the square of the altitude. For all measurements, the $57^{\circ}$ inclination of the station is sufficiently high to obtain full coverage of tropical and regions where cloud radiation forcing has its greatest impact. Mid-latitude regions are also covered. More significantly, the orbit precesses rapidly with respect to solar angles and thus there is sampling through the full diurnal cycle. Most

current and planned low earth orbit measurements for clouds and aerosol are from sun synchronous obits. The diurnal cycle is known to be a large factor, and there is a question on the sampling bias of current observations. A well know limitation of the space station as an earth observation platform is the lack of pointing stability. For active atmospheric sensors, the issue is to obtain good pointing knowledge rather than one of pointing accuracy. Pointing knowledge is not difficult to obtain. Pointing stability is a significant issue for imaging radiometers, but image stabilization mirrors and platforms for space instruments are a well developed technology. Likely a common system approach could be applied across all instruments.

\section{CONCLUSION}

The need for an earth science mission to study the full three dimensional cloud and aerosol structure and the associated physical parameters leading to a complete measurement of radiation forcing processes is well recognized. The mission requires combined observations from six or more specific instruments including active radar and lidar system. The international space station is a desirable platform for the mission due to the potential to significantly lower instrument costs and an appropriate orbital altitude and inclination.

\section{REFERENCES}

Brown, P. R., Illingworth, A. J., Heymsfield, A. J., Browning, K. A., and Gosset, M., J. Appl. Meteorol., 34, 2346$2366(1995)$

Spinhime, J. D., Hart, W. D. and Hlavka, D. L., J. Atmos. Sci., 53, 1438-1458 (1998) 